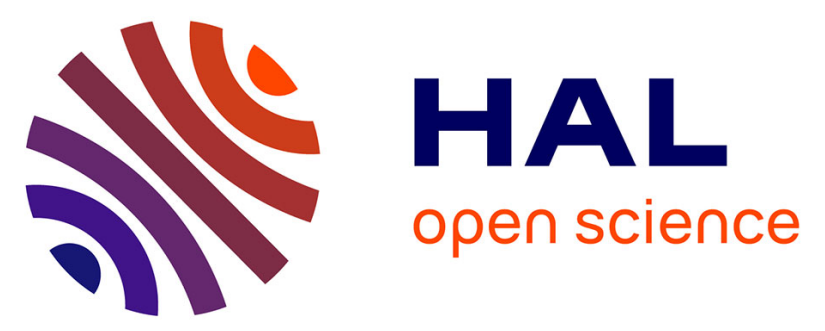

\title{
Relaxation of the Collective Magnetization of a Dense 3D Array of Interacting Dipolar $\mathrm{S}=3$ Atoms
}

\author{
Lucas Gabardos, Bihui Zhu, Steven Lepoutre, Ana Maria Rey, B
}

Laburthe-Tolra, Laurent Vernac

\section{To cite this version: \\ Lucas Gabardos, Bihui Zhu, Steven Lepoutre, Ana Maria Rey, B Laburthe-Tolra, et al.. Relaxation of the Collective Magnetization of a Dense 3D Array of Interacting Dipolar S = 3 Atoms. Physical Review Letters, 2020, 125 (14), 10.1103/PhysRevLett.125.143401 . hal-03064430}

\author{
HAL Id: hal-03064430 \\ https://hal.science/hal-03064430
}

Submitted on 14 Dec 2020

HAL is a multi-disciplinary open access archive for the deposit and dissemination of scientific research documents, whether they are published or not. The documents may come from teaching and research institutions in France or abroad, or from public or private research centers.
L'archive ouverte pluridisciplinaire HAL, est destinée au dépôt et à la diffusion de documents scientifiques de niveau recherche, publiés ou non, émanant des établissements d'enseignement et de recherche français ou étrangers, des laboratoires publics ou privés. 


\title{
Relaxation of the Collective Magnetization of a Dense 3D Array of Interacting Dipolar $S=3$ Atoms
}

\author{
Lucas Gabardos@, ${ }^{1,2, \dagger}$ Bihui Zhu, ${ }^{3,4, \dagger}$ Steven Lepoutre, ${ }^{1,7}$ Ana Maria Rey, ${ }^{5,6}$ \\ Bruno Laburthe-Tolra, ${ }^{2,1}$ and Laurent Vernac ${ }^{1,2 *}$ \\ ${ }^{1}$ Laboratoire de Physique des Lasers, Université Paris 13, F-93430 Villetaneuse, France \\ ${ }^{2}$ CNRS, UMR 7538, LPL, F-93430 Villetaneuse, France \\ ${ }^{3}$ ITAMP, Harvard-Smithsonian Center for Astrophysics, Cambridge, Massachusetts 02138, USA \\ ${ }^{4}$ Department of Physics, Harvard University, Cambridge, Massachusetts 02138, USA \\ ${ }^{5}$ JILA, NIST and Department of Physics, University of Colorado, Boulder, Colorado 80309, USA \\ ${ }^{6}$ Center for Theory of Quantum Matter, University of Colorado, Boulder, Colorado 80309, USA \\ ${ }^{7}$ Université Paris-Saclay, CNRS, Laboratoire Aimé Cotton, 91405, Orsay, France
}

(Received 26 May 2020; accepted 14 August 2020; published 30 September 2020)

\begin{abstract}
We report on measurements of the dynamics of the total magnetization and spin populations in an almost unit-filled lattice system comprising about $10^{4}$ spin $S=3$ chromium atoms, under the effect of dipolar interactions. The observed spin population dynamics is unaffected by the use of a spin echo and fully consistent with numerical simulations of the $S=3 X X Z$ spin model. On the contrary, the observed magnetization decays slower than in simulations and, surprisingly, reaches a small but nonzero asymptotic value within the longest timescale. Our findings show that spin coherences are sensitive probes to systematic effects affecting quantum many-body behavior that cannot be diagnosed by merely measuring spin populations.
\end{abstract}

DOI: 10.1103/PhysRevLett.125.143401

Synthetic atom-based materials are emerging as unique quantum laboratories for the exploration of collective behaviors in interacting many-body systems [1]. In particular, both electric and magnetic dipolar gases featuring long-range spin-spin interactions are opening great opportunities for the exploration of quantum magnetism in regimes inaccessible to gases interacting via purely contact interactions [2].

While electric dipolar interactions are fundamentally stronger and have led to important breakthroughs, as demonstrated by recent experiments using $\mathrm{KRb}$ molecules in 3D lattices [3] and Rydberg atoms in bulk gases [4], as well as in optical tweezers arrays [5-10], magnetic quantum dipoles offer complementary unique opportunities for quantum simulations. For example, they provide the possibility to trap low entropy and dense macroscopic arrays of $S>1 / 2$ atoms in close to unit-filled 3D optical lattice potentials where a truly collective many-body behavior manifests itself. Under these conditions, it is possible to study spin models with large spins [11-13], which cost exponentially more resources to classically simulate [14] than conventional $S=1 / 2$ models of magnetism. These capabilities have started to be explored in experiments working both with bosonic chromium and fermionic erbium atoms in 3D lattices [12,13,15-17], which have observed already signatures of rich many-body dynamics, including quantum thermalization and the buildup of many-body correlations. However, so far all the information has only been extracted from measurements of spin populations without direct access to quantum coherences, which contain key signatures of the underlying quantum dynamics $[3,4]$.

Here we make a step forward and report time-resolved measurements of the spin coherence and also populations of a many-body strongly interacting $S=3$ dipolar gas of ${ }^{52} \mathrm{Cr}$ atoms in a deep 3D lattice. The spin coherence is extracted from measurements of the collective transverse magnetization of the gas $J_{\perp}$ via Ramsey spectroscopy. Since the longitudinal magnetization remains zero at all times, the transverse magnetization is equal to the total magnetization (which is the norm of the collective spin) of the ensemble. The system is initially prepared in a spin coherent state with maximal transverse magnetization $J_{\perp}=N S$, which is let to evolve due to magnetic dipolar couplings. Our experimental protocol includes a spin-echo pulse at the middle of the dynamics to reduce the effect of magnetic field inhomogeneities.

In agreement with previous results [15-17], we find that the spin population dynamics is well captured by a semiclassical method, referred to as the generalized discrete truncated Wigner approximation (GDTWA), based on a Monte Carlo sampling in phase space $[18,19]$. In addition, we find that spin population dynamics is barely affected by the spin echo. However, we observe that the transverse magnetization decays at a slower rate than the one expected from numerical simulations based on a pure spin $X X Z$ 
model. In addition, the magnetization surprisingly saturates at a nonzero value in the experimentally investigated timescale. We attribute these discrepancies to effects not included in the pure spin model, such as tunneling induced by lattice heating. We provide toy model simulations that support this claim. Our observations highlight the relevance of quantum coherence to characterize many-body phenomena.

Our experimental platform differs from previous studies on the transverse magnetization of ensembles of dipolar particles $[3,4]$ in that it consists of a high density ordered array of $S=3$ spin particles. It is obtained by loading a ${ }^{52} \mathrm{Cr}$ Bose-Einstein condensate (BEC) in a 3D optical lattice deep into the Mott regime. The lattice implemented, made with five lasers at $\lambda_{L}=532 \mathrm{~nm}$, is a parallelepiped with periodicities of $(268,299,597) \mathrm{nm}$ and a total lattice depth of 90 recoil energy at $\lambda_{L}$. We estimate the tunneling time to be $\simeq 100 \mathrm{~ms}$. We obtain typically $N=10^{4}$ atoms close to unit filling (see [15] for more details). Initially the sample is prepared in a spin coherent state, with all spins in the maximally stretched state $m_{S}=-3$ and antialigned with the external magnetic field $\mathbf{B}$. Spin dynamics is triggered by rotating all spins at $t=0$ by $\pi / 2$, with the use of a resonant rf pulse [15] (see Fig. 1); the estimated accuracy on the rotation angle is $\pm 2 \%$. We measure the dynamical evolution of the seven spin populations $N_{m_{S}}$ in the basis set by the external magnetic field through Stern-Gerlach separation. We also probe the norm of the collective spin $\mathbf{J}=\sum_{i=1}^{N} \mathbf{S}_{i}$, which reads $\|\mathbf{J}\|=\sqrt{\left\langle J_{x}\right\rangle^{2}+\left\langle J_{y}\right\rangle^{2}+\left\langle J_{z}\right\rangle^{2}} ;$ this quantity, ranging from 0 to $3 N$, is equal to the total magnetization and is maximal for the initial spin state. In the following, we will use normalized quantities: $\mathbf{j}=\mathbf{J} / N$ and $\ell=\|\mathbf{j}\|$.

To measure $\ell$ we use a Ramsey protocol, in which a second $\pi / 2$ rotation is imparted just before population measurements (see Fig. 1). B is along $z$. In the rotating frame (turning around $z$ at the rf frequency), rf pulses ensure rotation of the spins around an axis called $y$. After the first $\pi / 2$ pulse, $\mathbf{j}_{t=0} \propto \hat{x}$. During the spin dynamics, fluctuations of the external magnetic field make $\mathbf{j}$ rotate in the $x y$ plane. We call $\phi(t)$ the angle between $\mathbf{j}$ and $\hat{x}$ and use it to define a new basis $X Y z$ where $\mathbf{j} \| \hat{X}$, i.e., $\ell=\left\langle\hat{j}_{X}\right\rangle$. All our data are based on the measurement of the component of the collective spin along the axis of the external magnetic field $z$ through a Stern-Gerlach separation. We call $M_{z}=\sum_{m_{S}} p_{m_{S}} m_{S}$ the normalized value of the measured spin component, with $p_{m_{S}}$ the fractional spin population of the $m_{S}$ component $\left(\sum_{m_{S}} p_{m_{S}}=1\right)$. Since the second $\pi / 2$ pulse again rotates spins around $y, M_{z}$ corresponds to a measurement of $\hat{j}_{x}=\cos (\phi) \hat{j}_{X}-\sin (\phi) \hat{j}_{Y}$ when using a Ramsey sequence. As $\phi(t)$ is different from trial to trial, we deduce the magnetization of the sample from statistical analysis (see below).

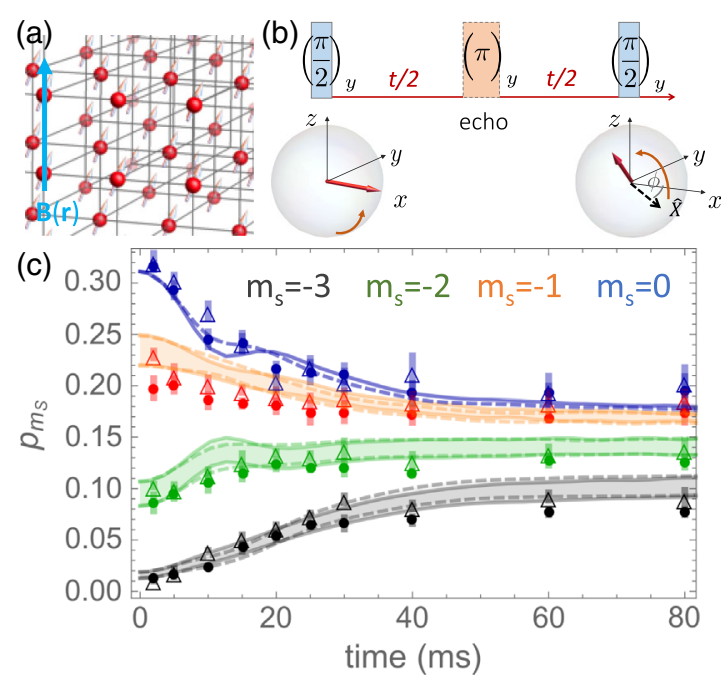

FIG. 1. (a) The experimental system consists of a 3D array of dipolar $\mathrm{Cr}$ atoms, with a spatially varying magnetic field $B(\mathbf{r})$. (b) Experimental rf sequence: $\mathrm{Cr}$ atoms are initialized in the $m_{s}=-3$ spin state and rotated by the first $\pi / 2$ pulse to align with the $x$ direction; the second $\pi / 2$ pulse is used to measure the magnetization. Because of magnetic noise, the collective spin makes an angle $\phi$ with respect to $x$ when this second pulse is imparted. We impart or not an echo pulse using an additional $\pi$ pulse at half the evolution time $t / 2$. (c) Time evolution of the fractional populations $p_{m_{s}}$ of four $m_{s}$ spin components, with and without spin echo (full circles and empty triangles, respectively). Each data point corresponds to the average of ten realizations. Error bars correspond to statistical uncertainties. The solid (dashed) lines show the numerical results obtained with GDTWA with (without) spin echo, for a lattice with unit filling and $B_{Q} / h=-4 \mathrm{~Hz}$. From bottom to top: $m_{s}=-3$ (black), $m_{s}=-2$ (green), $m_{s}=-1$ (orange), $m_{s}=0$ (blue). The colored bands in the GDTWA calculations account for a $\pm 2 \%$ experimental error in the first pulse area.

If one can neglect tunneling, the prepared ensemble of $N$ coupled spins, which are pinned at the individual sites of a 3D lattice, evolve under a pure spin model. The mean external magnetic field amplitude, $B_{0}=1.5 \mathrm{G}$, being strong enough to generate Zeeman splittings larger than nearest-neighbor dipolar interactions, the dynamics is described by the following $X X Z$ spin model [12]:

$$
\hat{H}_{d d}=\sum_{i>j}^{N} V_{i j}\left[\hat{S}_{i}^{z} \hat{S}_{j}^{z}-\frac{1}{2}\left(\hat{S}_{i}^{x} \hat{S}_{j}^{x}+\hat{S}_{i}^{y} \hat{S}_{j}^{y}\right)\right],
$$

where $\quad V_{i, j}=V_{d d}\left[\left(1-3 \cos ^{2} \theta_{i, j}\right) / r_{i, j}^{3}\right], \quad$ with $\quad V_{d d}=$ $\left\{\left[\mu_{0}\left(g \mu_{B}\right)^{2}\right] / 4 \pi\right\}, \mu_{0}$ as the magnetic permeability of vacuum, $g \simeq 2$ as the Landé factor, and $\mu_{B}$ as the Bohr magneton. The sum runs over all pairs of particles $(i, j) . r_{i, j}$ is the distance between atoms, $\theta_{i, j}$ is the angle between their interatomic axis and the external magnetic field, and $\hat{\mathbf{S}}_{i}=\left\{\hat{S}_{i}^{x}, \hat{S}_{i}^{y}, \hat{S}_{i}^{z}\right\}$ are spin-three angular momentum operators, associated with atom $i$. The strongest dipolar 
coupling is obtained from the shortest intersite distance $r_{\min }=268 \mathrm{~nm}$ in our lattice [15], and $V_{d d} / r_{\min }^{3} \simeq 3 \mathrm{~Hz}$. For an ensemble of dipolar spins, the normalized magnetization $\ell$ decreases as a result of interactions, which at short time reads

$$
\ell(t)=3-\frac{81 t^{2} V_{\mathrm{eff}}^{2}}{16 \hbar^{2}}
$$

where $V_{\text {eff }} / h=\sqrt{1 / N \times \sum_{i \neq j}^{N} V_{i j}^{2}} / h \simeq 6 \mathrm{~Hz}$ for a unitfilled lattice in our experiment. This leads to a typical timescale $\tau_{d d} \simeq 20 \mathrm{~ms}$ for $\ell$ to reach zero. This is a pure quantum effect since a mean-field ansatz predicts no decay $[15,20,21]$. We note that similar dipolar-induced magnetization decay and evidence of the buildup of multiple-spin coherences has been reported in NMR systems, where, however, the system starts in a highly mixed state [22].

In addition to $\hat{H}_{d d}$, atoms experience a tensor light shift $\hat{H}_{Q}=\sum_{i} B_{Q}\left(\hat{S}_{i}^{z}\right)^{2}$. For a noninteracting gas, this leads to a periodic evolution of $\ell$, with a timescale $\tau_{q}=\left[h /\left(4\left|B_{Q}\right|\right)\right] \approx 50 \mathrm{~ms}$ to reach zero for the first time, for a typical $\left|B_{Q}\right| / h \approx 5 \mathrm{~Hz}$ in our experiment. This one-body term has to be taken into account in simulations.

At short time, it leads to a replacement of $V_{\text {eff }} \rightarrow$ $V_{\text {eff }} \sqrt{1+40 B_{Q}^{2} / 27 V_{\text {eff }}^{2}}$ in Eq. (2).

Furthermore, magnetic field inhomogeneities described by gradients for the Larmor frequency, $\omega_{L, i}=$ $g \mu_{B} / \hbar\left(B_{0}+\vec{b} \cdot \vec{r}_{i}\right)$, lead to another term in the Hamiltonian, $\hat{H}_{B}=\sum_{i} \hbar \omega_{L, i}\left(\hat{S}_{i}^{z}\right)$, which generates dephasing and leads to a damping of $\ell$. The damping timescale is $\tau_{b}=\left[h / 2 g \mu_{B} b R\right] \simeq 3 \mathrm{~ms}$ with $b \simeq 13 \mathrm{Gm}^{-1}$, and $R \simeq 5 \mu \mathrm{m}$ is the typical size of the sample. In order to compensate for this dephasing, we implement a spin-echo technique, in which spins are rotated by $\pi$ in the middle of the dynamics (see Fig. 1).

One question that naturally arises is whether the spin echo changes as well the evolution of the population on different Zeeman levels. As shown in Fig. 1, the observed spin population dynamics is roughly identical with and without the echo, which is confirmed by GDTWA numerical simulations using the experimental gradient. This behavior is consistent with a short time perturbative analysis, which predicts that magnetic field gradients only enter at quartic order in the population dyna mics, i.e., $\propto\left(5 t^{4} / 256 N\right)\left\{15 \sum_{i=1}^{N}\left[\sum_{j \neq i}^{N} V_{i j}\left(\omega_{L i}-\omega_{L j}\right)\right]^{2}-\right.$ $\left.27 \sum_{i, j \neq i}^{N}\left(\omega_{L i}-\omega_{L j}\right)^{2} V_{i j}^{2}\right\}$, while dipolar effects enter at second order $\propto t^{2} V_{\text {eff }}^{2}$ (see Supplemental Material [23]).

Our raw experimental results to measure the magnetization are shown in Fig. 2. Without a spin echo, a fast damping of the measured values of $M_{z}$ is observed, in a timescale consistent with $\tau_{b}$. There is here a striking difference with our previous measurements in a bulk BEC [21], where a gap due to spin-dependent interactions
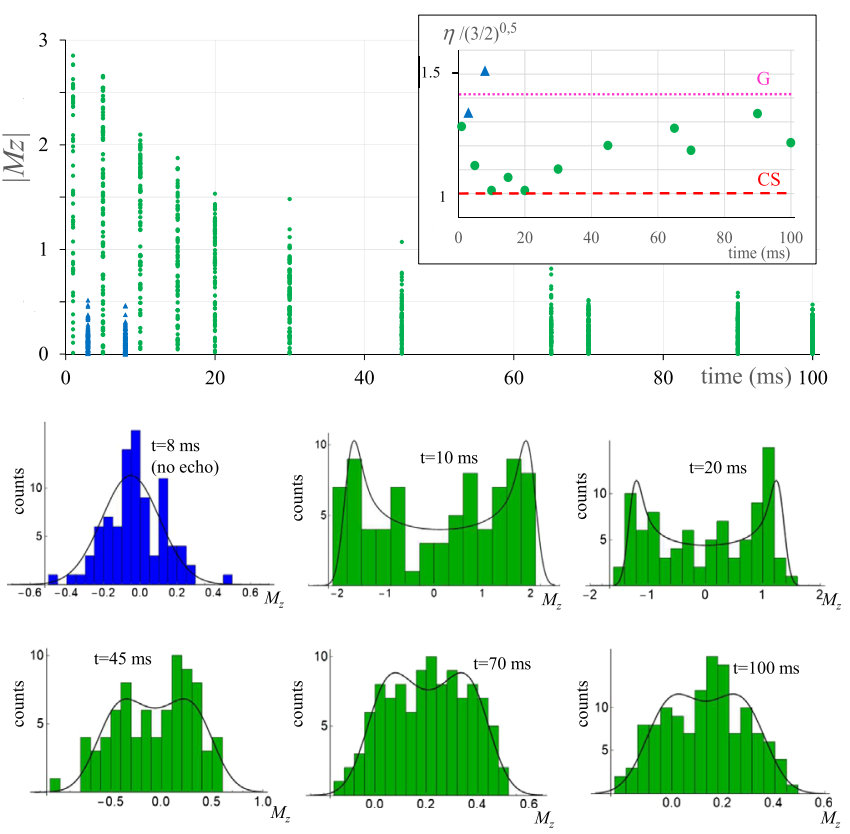

FIG. 2. Normalized spin component $M_{z}$ measured after a Ramsey sequence, with 60-100 realizations for each spin dynamics duration. Top: absolute values of $M_{z}$; blue triangles and green circles correspond to experiments without and with spin echo, respectively. Inset: the parameter $\eta$ (see text) is evaluated from the corresponding $M_{z}$ distributions. The horizontal lines show the expected value for respectively the PD of a classical spin (dashed, red) and the Gaussian PD (dotted, purple). Bottom: histograms of the $M_{z}$ distributions are shown together with the PD used to fit them (solid line); see text.

prevents the reduction of magnetization. When a spin echo is applied, the raw data show that $\ell$ decays with a significantly longer timescale, compatible with $\tau_{d d}$. We note that, given that $\hat{H}_{d d}$ does not commute with $\hat{H}_{B}$, the utility of a spin echo to protect the decay of $\ell$ is parameter and geometry dependent [25].

To obtain a quantitative estimate of $\ell$ as a function of time, we have investigated the probability distributions (PDs) associated with the data. Figure 2 shows that a mostly Gaussian PD is obtained for experiments without echo. On the contrary, data with spin echo only show a Gaussian-like shape at long times. At short time, PDs of a totally different kind are obtained, with a maximum of the probability for large values of $\left|M_{z}\right|$. To account for this observation, we introduce the probability distribution of a "classical spin" (CS), i.e., associated with a classical vector with norm $\ell$ and no fluctuations. The corresponding number $d n$ of realization of $M_{z}$ reads [23]

$$
\frac{d n}{d M_{z \mathrm{CS}}}=\frac{1}{\pi \ell} \frac{1}{\sqrt{1-\frac{M_{z}^{2}}{\ell^{2}}}} .
$$

In order to characterize the observed PDs, we evaluate from the data the square root of the kurtosis 
$\eta=\left(\sqrt{M_{4}} / M_{2}\right)$ with $M_{n}=\int \operatorname{PD}(x) x^{n} d x: \eta=\sqrt{\frac{3}{2}}$ for the PD of Eq. (3), and $\eta=\sqrt{3}$ for a Gaussian PD, $\left(d n / d M_{z}\right)_{G}=(1 / \sqrt{\pi} \sigma) \exp \left[-\left(M_{z}^{2} / \sigma^{2}\right)\right]$. The experimental values of $\eta$ are shown in Fig. 2. Data without echo show a good agreement with a Gaussian PD. For data with a spin echo, the value of $\eta$ is in good agreement with the classical value for $t_{f} \simeq 5-30 \mathrm{~ms}$, and it gradually approaches a Gaussian value for $t>60 \mathrm{~ms}$. This first qualitative analysis shows trends for the measured PDs. In order to get numerical values of $\ell$, we have used a convolution of the two PDs described above to fit the data, as shown in Fig. 2; this method assumes that a total dephasing has occurred, which requires $t \geq 10 \mathrm{~ms}$ in our experiment (for $t=1,5 \mathrm{~ms}$, see the Supplemental Material [23]).

The corresponding results of $\ell$ for each time $t$ are shown in Fig. 3. As expected, without applying the echo pulse, $\ell$ decays rapidly; the actual damping rate depends on the system size and lattice geometry. On the other hand, the measured data of $\ell$ after applying the echo pulse reveal an exponential damping of the magnetization toward a small but not zero value, $\ell(t) \simeq\left(3-\ell_{0}\right) \exp \left(-t / \tau_{e}\right)+\ell_{0}$, with $\ell_{0}=0.15$ and $\tau_{e}=22 \mathrm{~ms}$. This contrasts with the glassy dynamics observed in, e.g., Ref. [4].

In order to model the dynamics of $\ell$ in the absence of the echo pulse, it is crucial to appropriately account for the actual sample geometry in experiment and to capture

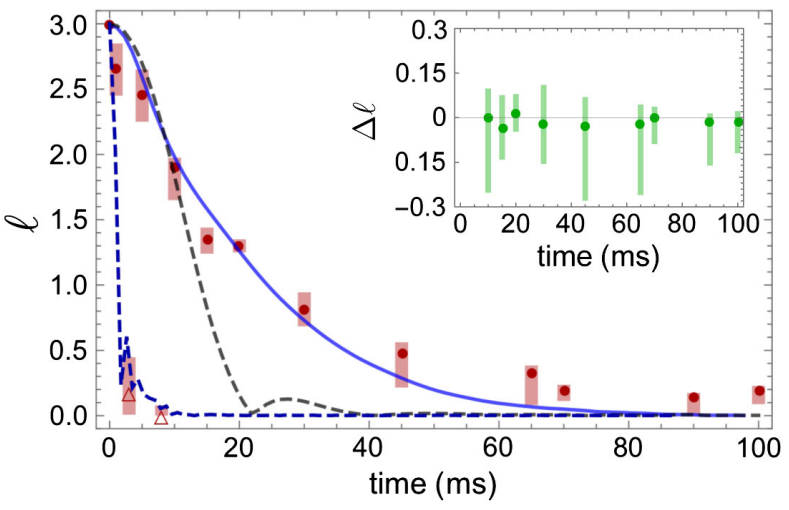

FIG. 3. Values of the normalized magnetization $\ell$ derived by fitting the distributions of $M_{z}$ after the Ramsey sequence. Error bars represent the $68 \%$ confidence interval and are detailed in the Supplemental Material [23]. Filled circles and empty triangles are measurements with and without the spin-echo pulse, respectively. The black dashed line shows the numerical results with the spinecho pulse applied, obtained with GDTWA for the same lattice configuration as in Fig. 1. The blue dashed line shows the dynamics without the spin echo, obtained from the Gaussian TWA simulations [23]. The blue solid line corresponds to GDTWA simulations effectively accounting for atomic motion in the lattice, with $B_{Q} / h=-2 \mathrm{~Hz}$. Inset: difference between the two experimental determinations of $\ell$, comparing the results of the fit of the experimental probability distributions to Eq. (5). Error bars correspond to the quadratic average of the standard deviations associated with either methods. the effects of inhomogeneities. For this purpose, we implement a Gaussian truncated Wigner approximation (TWA) approach in our numerical calculation (blue dashed line in Fig. 3) $[23,26]$, which allows for efficiently simulating systems with $N \sim 10^{4}$, much larger than the size previously investigated [15]. When the echo pulse is applied, we first use GDTWA simulations with the same parameters as those used in Fig. 1. We explicitly insert a $\pi$ rotation around the $y$ axis at half of the evolution time (see Supplemental Material [23]). While the GDTWA captures the population dynamics at all times (see Fig. 1), it is only able to reproduce the magnetization measurements at $t \leq 10 \mathrm{~ms}$ (black dashed line in Fig. 3). Interestingly, the magnetization dynamically evolves for $t>30 \mathrm{~ms}$, whereas the population dynamics and pure spin model numerical simulations have then essentially reached a plateau. This indicates that measuring the magnetization constitutes a more sensitive probe than simply monitoring spin population dynamics.

While tunneling in the lowest band (where atoms are initially loaded) is too slow to explain the discrepancy between the magnetization data and the GDTWA simulations, one possibility could be that phase noise in the lattice could promote particles to higher bands, where tunneling is non-negligible. This type of heating process was, for example, also reported with KRb molecules [27]. To model this possible scenario, we performed numerical simulations assuming frozen atoms but relaxing the requirement for them to be pinned in the regular grid imposed by the lattice potential, while keeping the same average density [23]. This emulates the idea that, during a tunneling process, on average, an atom can be in between two adjacent lattice sites. The calculated dynamics of spin populations resulting from this toy model is consistent with the experimental measurements (see the Supplemental Material [23]). The result for $\ell$ is shown with a solid line in Fig. 3: the agreement with the experimental data is much better than the one obtained with GDTWA (black dashed line). However, our toy model predicts a zero relaxation value of the magnetization within the experimental time range investigated, in contrast with the experimental observations.

In order to confirm our measurements of $\ell$, we performed a noise analysis of the components of the collective spin. Whether we apply the final $\pi / 2$ pulse (Ramsey experiment, labeled R) or not (labeled noR), we measure $\hat{j}_{x}$ or $\hat{j}_{z}$. Taking into account the randomness of $\phi$ [28], one obtains the following expressions for the variance of $M_{z}$ when averaging over many realizations:

$$
\begin{aligned}
\operatorname{Var}\left(M_{z}\right)_{\mathrm{R}} & =\frac{\ell^{2}}{2}+\frac{\operatorname{Var}\left(\hat{j}_{X}\right)+\operatorname{Var}\left(\hat{j}_{Y}\right)}{2}+\sigma_{\mathrm{exp}}^{2} \equiv\left\langle j_{x}^{2}\right\rangle_{\mathrm{exp}}, \\
\operatorname{Var}\left(M_{z}\right)_{\mathrm{noR}} & =\frac{3}{2 N}+\sigma_{\mathrm{exp}}^{2} \equiv\left\langle j_{z}^{2}\right\rangle_{\mathrm{exp}} .
\end{aligned}
$$

To derive Eq. (4) we add a technical noise (with an associated standard deviation $\sigma_{\text {exp }}$ ) to the theoretical 
expectations. We assume that measurements of $\hat{j}_{z}$ and $\hat{j}_{x}$ are affected by the same technical noise (an insufficient signal to noise ratio in the absorption images). Since $\left[\hat{H}_{d d}, \hat{J}_{z}\right]=0$, the theoretically expected value of $\left\langle\hat{j}_{z}^{2}\right\rangle$ remains equal to its value at $t=0$, i.e., to the standard quantum noise (SQN) $3 / 2 N$.

The experimental standard deviation without Ramsey pulse is about 3 times the SQN for $t=0$ and gets larger as $t$ increases [23]. We thus consider that $\left\langle j_{z}^{2}\right\rangle_{\exp } \simeq \sigma_{\exp }^{2}$. In our simulations for the quantum noise of $\hat{j}_{x}$ [23], we get $\sigma_{\text {exp,min }}^{2}>4 \times\left\{\left[\operatorname{Var}\left(\hat{j}_{X}\right)+\operatorname{Var}\left(\hat{j}_{Y}\right)\right] / 2\right\}_{\max } \simeq(32 / N)$. We therefore assume that $\left\langle j_{x}^{2}\right\rangle_{\exp } \simeq\left(\ell^{2} / 2\right)+\sigma_{\exp }^{2}$ and get

$$
\left\langle j_{x}^{2}\right\rangle_{\exp } \simeq \frac{\ell^{2}}{2}+\left\langle j_{z}^{2}\right\rangle_{\exp } .
$$

When the dephasing is complete, Eq. (5) provides a second method for measuring $\ell$, which is in good agreement with the first method described above (see inset of Fig. 3).

Obtaining a coherence time longer than expected from frozen particles models is unusual. It brings up the interesting observation that motion is not always a detrimental effect, which has been learned in NMR (motional narrowing, see [29]). In our case, the scenario of quantum thermalization links the damping of the collective spin to the growth of entanglement. Motion generates disorder in the lattice, which may disrupt the growth of entanglement generated by dipolar couplings and slow down thermalization, hence the slower decay of $\ell$.

In conclusion, our experiment demonstrates the remarkably different effects of a spin echo on the dynamics of a strongly interacting quantum system for spin populations and spin magnetization. Notably, our measurements show that the decay of the magnetization in our experiment is slower than expected by a spin model of frozen particles, a finding that could not be previously deduced from the measurements on population dynamics. This illustrates how measurements of spin coherences provide valuable information on quantum many-body systems that are crucial to benchmarking experiments as quantum simulators.

We thank Benoit Darquié, Tommaso Roscilde, and Johannes Schachenmayer for useful discussions and Thomas Bilitewski and Itamar Kimchi for reviewing the manuscript. The Villetaneuse group acknowledges financial support from CNRS, Université Sorbonne Paris Cité (USPC), Conseil Régional d'Ile-de-France under Sirteq Agency, the Indo-French Centre for the Promotion of Advanced Research-CEFIPRA under the LORIC54041 contract, Agence Nationale de la Recherche (Project No. ANR-18-CE47-0004), and QuantERA ERA-NET (MAQS project). A.M.R is supported by the AFOSR Grant No. FA9550-18-1-0319, by the DARPA DRINQs grant, the ARO single investigator Grant No. W911NF-191-0210, the NSF PHY1820885, NSF JILA-PFC PHY-1734006 grants, and by NIST. B.Z. is supported by the NSF through a grant to ITAMP.

* Corresponding author.

laurent.vernac@univ-paris13.fr

These authors contributed equally to this work.

[1] C. Gross and I. Bloch, Science 357, 995 (2017).

[2] J. L. Bohn, A. M. Rey, and J. Ye, Science 357, 1002 (2017).

[3] B. Yan, S. A. Moses, B. Gadway, J. P. Covey, K. R. A. Hazzard, A. M. Rey, D. S. Jin, and J. Ye, Nature (London) 501, 521 (2013).

[4] A. Signoles, T. Franz, R. F. Alves, M. Gärttner, S. Whitlock, G. Zürn, and M. Weidemüller, arXiv:1909.11959.

[5] H. Bernien, S. Schwartz, A. Keesling, H. Levine, A. Omran, H. Pichler, S. Choi, A. S. Zibrov, M. Endres, M. Greiner, V. Vuleti, and M. D. Lukin, Nature (London) 551, 579 (2017).

[6] A. Browaeys and T. Lahaye, Nat. Phys. 16, 132 (2020).

[7] S. de Léséleuc, V. Lienhard, P. Scholl, D. Barredo, S. Weber, N. Lang, H. P. Büchler, T. Lahaye, and A. Browaeys, Science 365, 775 (2019).

[8] P. Schauß, J. Zeiher, T. Fukuhara, S. Hild, M. Cheneau, T. Macrı, T. Pohl, I. Bloch, and C. Gross, Science 347, 1455 (2015).

[9] P. Schau, M. Cheneau, M. Endres, T. Fukuhara, S. Hild, A. Omran, T. Pohl, C. Gross, S. Kuhr, and I. Bloch, Nature (London) 491, 87 (2012).

[10] A. Keesling, A. Omran, H. Levine, H. Bernien, H. Pichler, S. Choi, R. Samajdar, S. Schwartz, P. Silvi, S. Sachdev, P. Zoller, M. Endres, M. Greiner, V. Vuleti, and M. D. Lukin, Nature (London) 568, 207 (2019).

[11] T. Lahaye, C. Menotti, L. Santos, M. Lewenstein, and T. Pfau, Rep. Prog. Phys. 72, 126401 (2009).

[12] A. de Paz, A. Sharma, A. Chotia, E. Maréchal, J. H. Huckans, P. Pedri, L. Santos, O. Gorceix, L. Vernac, and B. Laburthe-Tolra, Phys. Rev. Lett. 111, 185305 (2013).

[13] A. de Paz, P. Pedri, A. Sharma, M. Efremov, B. Naylor, O. Gorceix, E. Maréchal, L. Vernac, and B. Laburthe-Tolra, Phys. Rev. A 93, 021603(R) (2016).

[14] S. Hallgren, D. Nagaj, and S. Narayanaswami, Quantum Inf. Comput. 13, 721 (2013), https://dblp.org/db/journals/qic/ qic13.html.

[15] S. Lepoutre, J. Schachenmayer, L. Gabardos, B. H. Zhu, B. Naylor, E. Maréchal, O. Gorceix, A. M. Rey, L. Vernac, and B. Laburthe-Tolra, Nat. Commun. 10, 1714 (2019).

[16] P. Fersterer, A. Safavi-Naini, B. Zhu, L. Gabardos, S. Lepoutre, L. Vernac, B. Laburthe-Tolra, P. B. Blakie, and A. M. Rey, Phys. Rev. A 100, 033609 (2019).

[17] A. Patscheider, B. Zhu, L. Chomaz, D. Petter, S. Baier, A.-M. Rey, F. Ferlaino, and M. J. Mark, Phys. Rev. Research 2, 023050 (2020).

[18] B. Zhu, A. M. Rey, and J. Schachenmayer, New J. Phys. 21, 082001 (2019).

[19] J. Schachenmayer, A. Pikovski, and A. M. Rey, Phys. Rev. X 5, 011022 (2015). 
[20] Y. Kawaguchi, H. Saito, and M. Ueda, Phys. Rev. Lett. 98, 110406 (2007).

[21] S. Lepoutre, K. Kechadi, B. Naylor, B. Zhu, L. Gabardos, L. Isaev, P. Pedri, A. M. Rey, L. Vernac, and B. LaburtheTolra, Phys. Rev. A 97, 023610 (2018).

[22] H. Cho, T. D. Ladd, J. Baugh, D. G. Cory, and C. Ramanathan, Phys. Rev. B 72, 054427 (2005).

[23] See Supplemental Material at http://link.aps.org/ supplemental/10.1103/PhysRevLett.125.143401 for extended data and details on experimental procedure and simulations, which includes Ref. [24].

[24] R. Mukherjee, T. C. Killian, and K. R. A. Hazzard, Phys. Rev. A 94, 053422 (2016).
[25] C. Solaro, A. Bonnin, F. Combes, M. Lopez, X. Alauze, J.-N. Fuchs, F. Piéchon, and F. Pereira Dos Santos, Phys. Rev. Lett. 117, 163003 (2016).

[26] Note that, in this Letter, the Gaussian TWA approach is only used for finding the time evolution of $\ell$ without the echo pulse (Fig. 3, blue dashed line); the other results are obtained with GDTWA. Also see [23] for more details.

[27] A. Chotia, B. Neyenhuis, S. A. Moses, B. Yan, J. P. Covey, M. Foss-Feig, A. M. Rey, D. S. Jin, and J. Ye, Phys. Rev. Lett. 108, 080405 (2012).

[28] B. Lücke, J. Peise, G. Vitagliano, J. Arlt, L. Santos, G. Tóth, and C. Klempt, Phys. Rev. Lett. 112, 155304 (2014).

[29] E. Yablonovitch, Nature (London) 550, 458 (2017). 